\title{
The present situation of the disabled sports competitions in china
}

\author{
Lan Zhang \\ Department of P.E and Art Education, Zhejiang YueXiu University of Foreign Languages, Shaoxing 312000, China
}

\begin{abstract}
Less against China in participating paralympic sports for the disabled, the number of entries, the inexperienced and compete more than novice " 3 little more than a" status quo. Through time series analysis, this paper concludes that Chinese cities disabled athletes people, places and the number of the national fitness rehabilitation projects of provincial level rise over time, and municipal activities present circulation or seasonal changes over time. Therefore, the activities of municipal disabled persons need to strengthen management, to make it better serve the disabled, and then optimize the Chinese disabled person sports competition management mode, make it better manage local disabled persons' federation.
\end{abstract}

\section{Introduction}

Disabled Chinese number more than 8000 , became the largest number of people with disabilities around the world. Disabled people to participate in sports, not only can make the disabled people's psychological and emotional adjustment, but also make the best means to rehabilitation for the disabled, as well as to the whole society to show this group, with an unyielding spirit of a platform. Projects by participating in competitive sports for the disabled, showing their value and position, at the same time also make more disabled people to participate in the sports. China is also through the development of sports for the disabled to improve the satisfaction of the Chinese public. The development of competitive sports for the disabled in China compared with western developed countries, started relatively late, foundation is still very weak. And less in China in the paralympics entries, the number of entries, the inexperienced and compete more than novice " 3 little more than a" status quo [1-4].

From 08 Beijing paralympic games held, is the cause of China's athletic sports for the disabled to achieve a successful leap, the Chinese paralympic athletes performance is outstanding, achievement is ideal, this also from the side reflects the development level of China's sports for the disabled cause in constant increase. The disabled can play basketball, this sounds like a fable [5]. But today, in the flexible paralympic movement of the technology of the basketball players really let a person admire. In control under the condition of the wheelchair, while also line dribbling, turned around, and even shot, all of the same action, having an organized effort, make people sit up and take notice. Paralympics in Beijing, there is a classic basketball games for the disabled, against the United States is Canada. In the normal competition, there must be no suspense, the U.S. men's basketball team will certainly sweeping trounced the Canada. But in the real see paralympic game, in Canada team has a character, known as the "wheelchair Jordan", the overall strength makes Canada obvious jump, and the United States played two overtime, in the end, the Canadian team has finally entered the final victory over the United States [6-10]. There are no losers in the game, they are doing their best, the audience of the game is also very satisfied, really enjoy the process sometimes is more important than the result. Here, I want to those who "handicap will not" athletes said sincere regards and respect.

For this, the analysis of the China disabled persons to participate in sports, some of the statistical data of time series analysis, it is concluded that the development of Chinese sports for the disabled, and give reasonable Suggestions.

\section{Establishment of model}

For time series analysis, this article is in accordance with the time changes to list events down successively, and constitutes an analysis of time series. We make an observation and study of time series effectively, then find out its corresponding change and law of development, finally we predict that its future trend is corresponding to the time series analysis.

First, we need understand the following model for time series analysis.

$$
\begin{aligned}
& \text { (1) Mode AR (p): } \\
& \left\{\begin{array}{l}
x_{t}=\Phi_{0}+\Phi_{1} x_{t-1}+\Phi_{2} x_{t-2}+\ldots+\Phi_{p} x_{t-p}+\varepsilon_{t} \\
\Phi_{p} \neq 0 \\
E\left(\varepsilon_{t}\right)=0, \quad \operatorname{Var}\left(\varepsilon_{t}\right)=\sigma_{\delta}^{2}, E\left(\varepsilon_{t} \varepsilon_{s}\right)=0, s \neq t \\
E x_{s} \varepsilon_{t}=0, \forall s<t
\end{array}\right.
\end{aligned}
$$

a Corresponding author: LanZhang44@sina.com 
The mode of the above structure is called auto-regressive model of $p$ order, recorded as AR(p).

(2) Mode $\operatorname{MA}(q)$ :

$$
\left\{\begin{array}{l}
x_{t}=u+\varepsilon_{t}-\theta_{1} \varepsilon_{t-1}-\theta_{2} \varepsilon_{t-2}-\ldots-\theta_{q} \varepsilon_{t-q} \\
\theta_{q} \neq 0 \\
E\left(\varepsilon_{t}\right)=0, \operatorname{Var}\left(\varepsilon_{t}\right)=\sigma_{\delta}^{2}, E\left(\varepsilon_{t} \varepsilon_{s}\right)=0, s \neq t
\end{array}\right.
$$

The mode of the above structure is called auto-regressive model of $\mathrm{q}$, recorded as MA (q).

(3) Mode ARMA(p,q):

$$
\left\{\begin{array}{l}
x_{t}=\Phi_{0}+\Phi_{1} x_{t-1}+\Phi_{2} x_{t-2}+\ldots+\Phi_{p} x_{t-p}+\varepsilon_{t}-\theta_{1} \varepsilon_{t-1}-\theta_{2} \varepsilon_{t-2}-\ldots-\theta_{q} \varepsilon_{t-q} \\
\Phi_{p} \neq 0, \theta_{q} \neq 0 \\
E\left(\varepsilon_{t}\right)=0, \operatorname{Var}\left(\varepsilon_{t}\right)=\sigma_{\delta}^{2}, E\left(\varepsilon_{t} \varepsilon_{s}\right)=0, s \neq t \\
E x_{s} \varepsilon_{t}=0, \forall s<t
\end{array}\right.
$$

The mode of the above structure is called auto-regressive model of $p \mathrm{q}$, recorded $\operatorname{as} \operatorname{ARMA}(p, q)$.

\subsection{Modeling of stationary series}

(1) The process:

According to the number of volleyball papers in the sports core journals from 2008-2013this 6 years, we list the initial data of the following process (The correlation coefficient calculation sample), (Model identification), (Estimating numbers), (Model test), (Model optimizing), (Series forecast), (Model process).

(2) Calculate the correlation coefficient of the sample: Sample autocorrelation coefficient:

$$
\hat{\rho}_{k}=\frac{\sum_{t=1}^{n-k}\left(x_{t}-\bar{x}\right)\left(x_{t+k}-\bar{x}\right)}{\sum_{t=1}^{n}\left(x_{t}-\bar{x}\right)^{2}}
$$

Partial autocorrelation coefficient of samples:

$$
\hat{\Phi}_{k k}=\frac{\hat{D}_{k}}{\hat{D}}
$$

(3) Model identification

(4) The approximate distribution of the sample correlation coefficient:

Barlett:

$$
\hat{\rho}_{k} \sim N\left(0, \frac{1}{n}\right), n \rightarrow \infty
$$

Quenouille:

$$
\hat{\Phi}_{k k} \sim N\left(0, \frac{1}{n}\right), n \rightarrow \infty
$$

(5) Parameter estimation:

solve-for parameter is $p+q+2$ The commonly estimating method of unknown parameters is: Torque estimation, the maximum estimation, and significant test of mode 6

Purpose: test the validity of the model (to extract the sufficient of the information)

Inspection objects: the residual sequence
Judging principle: a good fitting model should be able to extract the sample observations in the sequence of related information, the residual sequence can discuss the data, and on the other hand, it needs to points the condition.

The original assumption: The number of volleyball papers on sports core journals.

$$
H_{0}: \rho_{1}=\rho_{2}=\ldots=\rho_{m}=0, \forall m \geq 1
$$

The alternative hypothesis: the corresponding residual is the differential data that need to be discussed.

test statistics:

$$
H_{1}: \rho_{k} \neq 0, \forall m \geq 1, k \leq m
$$

$$
\text { LB statistics: } L B=n(n+2) \sum_{k=1}^{m}\left(\frac{\hat{\rho}_{k}^{2}}{n-k}\right) \sim \chi^{2}(m)
$$

Parametric test of significance

Purpose: test each of the corresponding unknown parameter value is whether nonzero or not, delete the dominant parameter.

$$
H_{0}: \beta_{j}=0 \quad H_{1}: \beta_{j} \neq 0
$$

test statistics:

$$
T=\sqrt{n-m} \frac{\hat{\beta}_{j}-\beta_{j}}{\sqrt{a_{j j} Q(\widetilde{\beta})}} \sim t(n-m)
$$

Model optimization:

Questions: here, we assume that the model passed the corresponding inspection, it means the data is on a certain feasible range, this model can effectively draw the results of the corresponding optimization

Purpose: select the relatively optimal model.

Sequence prediction:

Linear prediction function: $x_{t}=\sum_{i=0}^{\infty} C_{i} x_{t-1-i}$

Variance

minimum

principle: $\operatorname{Var}_{\hat{x}_{t(l)}}\left[e_{t}(l)\right]=\min \left\{\operatorname{Var}\left[e_{t}(l)\right]\right\} \quad$ as the factors of the following model analysis.

\subsection{Non-stationary sequence modeling}

First we need the smooth operation of the data, then establish the model ARIMA (p, q) and calculate it by the model, we introduce the mode in the following:

The using occasions of the model structure is fitting difference stationary series.

Model structure: $\left\{\begin{array}{l}\Phi(B) \nabla^{d} x_{t}=\Theta(B) \varepsilon_{t} \\ E\left(\varepsilon_{t}\right)=0, \operatorname{Var}\left(\varepsilon_{t}\right)=\sigma_{\varepsilon}^{2}, E\left(\varepsilon_{t} \varepsilon_{s}\right)=0, s \neq t \\ E x_{s} \varepsilon_{t}=0, \forall s<t\end{array}\right.$

The process of establishing the model: (obtaining the observed sequence value, stationary test, difference operation, variables test, fitting ARMA model, analysis result).

\subsection{The application of the model and the corresponding data processing}

For the China disabled people's understanding of the most 
important thing of health problems, we make statistics from the survey of 501 people and we can know the concern of the disabled are: diet, health care, physical exercise these aspects. The importance of it is shown in Table 1.

Table 1. Disabled people's understanding of the most important thing of health problems

\begin{tabular}{ccc}
\hline $\begin{array}{c}\text { Disabled people's understanding } \\
\text { of the most important thing of } \\
\text { health problems }\end{array}$ & Number & Percentage \\
\hline Diet structure & 95 & $18.90 \%$ \\
Health care & 280 & $55.90 \%$ \\
Physical exercise & 126 & $25.20 \%$ \\
\hline
\end{tabular}

(Source: Cai Muyong etc. the results of the questionnaire)

It can be seen from the above analysis, as for the physical health of Chinese disabled people, the most one they focus on were the health care, $25.2 \%$ of people pay attention to physical exercise, which is relatively small, so cause that the base of the disabled in China is large, but overall the number of people to participate in sports can not increase.

Table 2. The purpose of the Chinese disabled people to participate in national fitness sports.

\begin{tabular}{ccc}
\hline Purpose & Number & Percentage \\
\hline Strong the body & 311 & $62.10 \%$ \\
Rehabilitate disease & 158 & $31.50 \%$ \\
Entertainment & 130 & $26.00 \%$ \\
Social interaction & 69 & $13.70 \%$ \\
Free-standing self-improvement & 93 & $18.50 \%$ \\
Reflect their own value & 46 & $9.10 \%$ \\
To relieve stress & 115 & $22.90 \%$ \\
Other & 44 & $8.80 \%$ \\
\hline
\end{tabular}

(Source: Cai Muyong etc. the results of the questionnaire)

We discuss the purpose of Chinese disabled people's participating in the national fitness movement, including (to keep fit, to disease rehabilitation, entertainment, social intercourse, self-reliant, manifest its value, relieve pressure, other) and carry on the data analysis. The statistical results are shown in Table 2.

It can be seen from the above analysis that Chinese disabled people's participating in national fitness movement mainly focused on the purpose of strengthening the body, which occupied $32 \%$ of the total. This shows that the purpose of Chinese disabled people's participating in physical exercise is very clear. Chinese disabled people's cultural level is generally low, the record of formal schooling of above of university is less than $1 \%$, and are mostly in the illiterate or semiliterate state. So we suggest that our government should pay more attention to the education of disabled people through the above survey, which can make Chinese disabled people receive a good education. The main factors that influencing the Chinese disabled people's participating in the activities of the national fitness analysis.

Corresponding to the above analysis, the main factors that influencing Chinese disabled people's participating in the activities of the national fitness is " unable to move freely" which occupied $35 \%$ of proportion as the whole. The next is $5^{\text {th }}$, "there is suitable exercise equipment, the sports place for the disabled, and then is $9^{\text {th }}$, "lack of professional guidance, consultancy, and specialized books". $10^{\text {th }}$ : no organization of exercise for the disabled, $13^{\text {th: }}$ : there is no time, they respectively account for $25.5 \%$ of the total, $24.1 \%, 28.3 \%$ and $26.7 \%$. Only $2.80 \%$ of people think that the problem is the unsupported of the family.

The index statistics of Chinese disabled population in recent five years and the statistical results of governments' fitness programs for the disabled. It is shown in Table 3.

Table 3. The index statistics of Chinese disabled population in recent five years.

\begin{tabular}{ccccc}
\hline Time & $\begin{array}{c}\text { Activities at the } \\
\text { provincial level } \\
\text { (place) }\end{array}$ & $\begin{array}{c}\text { Activities at } \\
\text { municipal level } \\
\text { (places) }\end{array}$ & $\begin{array}{c}\text { The national fitness } \\
\text { rehabilitation projects (a) }\end{array}$ & $\begin{array}{c}\text { Cities disabled athletes } \\
(\mathrm{m})\end{array}$ \\
\hline 2009 & 113 & 776 & 48 & 24069 \\
2010 & 126 & 629 & 40 & 30523 \\
2011 & 142 & 1455 & 56 & 25935 \\
2012 & 156 & 709 & 75 & 27879 \\
2013 & 169 & 857 & 80 & 49507 \\
\hline
\end{tabular}

(Source: Cai Muyong etc. the results of the questionnaire)

Corresponding to the above data and combined with the disabled people's understanding of the most important question of health problems, the purpose of Chinese disabled people to participate in national fitness movement, the comparison of the structure of the disabled culture degree, we carry on the time series analysis of the main factors that influencing the Chinese disabled people's participating in the activities of the national fitness and so on these factors. It is shown in Figure 1.
From the above data and time analysis, we can see the regulation that the activities at the provincial level, municipal activities, and the number of national rehabilitation exercise, cities disabled athletes; all of these have changed over time.

According to the time series analysis we know that pink line represents tendency, the green line represents seasonal cycle, the blue line represents stochastic. It is shown in the following Figure 2: 


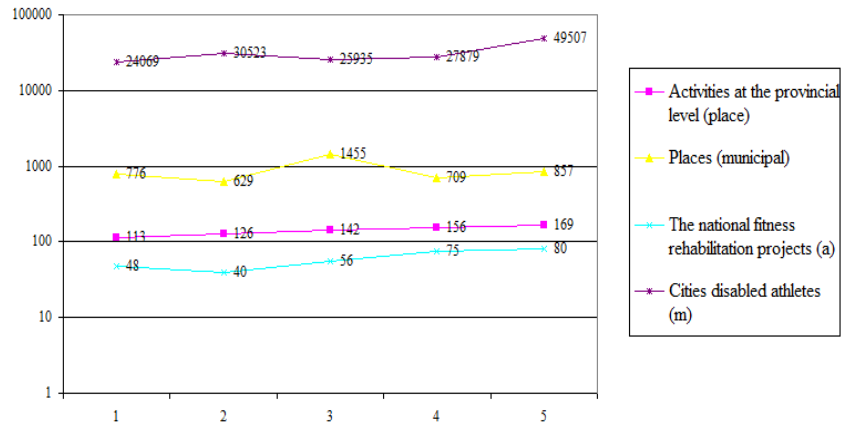

Figure 1. Time according to the sequence analysis results.

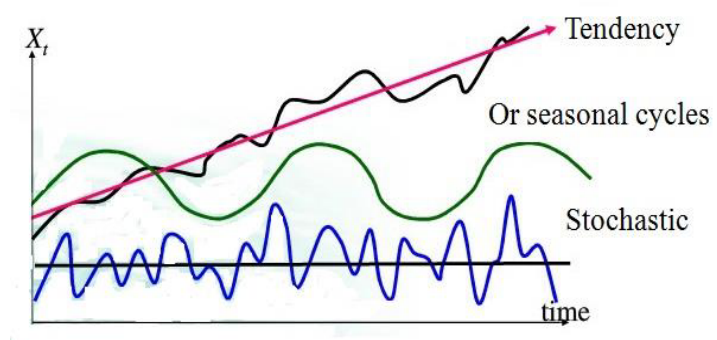

Figure 2. The decomposition of the time series data contrast figure.

According to Figure 1 and combined with Figure 2, we can see that cities disabled athletes , the activities at the provincial level and the number of national rehabilitation exercise have an increasing tendency with the change of time, however the municipal activities change with the seasonal cycles.

\subsection{Results analysis}

Through the above time sequence analysis results, we can find that the management of Chinese current municipal activities need to be strengthened, to make it better serve the disabled, we should optimize Chinese disabled sports competition management mode in view of the above situation, including CDPF, Group of the state sport general administration department, China's Olympic sports management center for the disabled, National team, Different provinces DPF, the training base.

\section{Conclusions}

The development of competitive sports for the disabled in China compared with western developed countries, started relatively late, foundation is still very weak. And less in China in the paralympics entries, the number of entries, the inexperienced and compete more than novice "3 little more than a" status quo.

Aimed at above present situation we analysis: disabled people's understanding of health the most important question, the purpose of Chinese disabled people to participate in national fitness movement, the structure of the disabled culture degree and the comparison, the main factors influencing the China disabled people to participate in the activities of the national fitness and so on. Through these conclusions: China is on the city's activities need to strengthen management, to make it better serve the disabled, to the Chinese disabled person sports competition management model optimization.

\section{References}

1. J.M. Xing, Y. Jiang, W.X. Yuan, L.Q. Liu, International Journal of Applied Mathematics and Statistics. 48, 461-468 (2013).

2. B. $\mathrm{Wu}, \mathrm{Z}$. Liu, Nature Environment and Pollution Technology. 15, 16-21 (2016).

3. D.Y. Tang, G. Ma, J. Guo, Information Technology Journal. 12, 3315-3319 (2013).

4. B. Zhang, K.L. Qin, L.B. Yu, International Journal of History and Cultural Studies. 2, 1-5 (2016).

5. X.Y. Zhang, Journal of Mechanical Engineering Research and Developments. 39, 217-225 (2016).

6. B. Zhang, K.L. Qin, X.F. Cao, International Journal of Sports and Physical Education. 2, 6-12 (2016).

7. W.Q. Jiang, K.L. Qin, B. Zhang, International Journal of Sports and Physical Education. 2, 12-15 (2016).

8. H.W. Eng, S.C. Ngien, Y.L. Hui, International Journal of Sports and Physical Education. 2, 1-7 (2016).

9. J. Zhang, H. Kazerooni, Journal of Mechanical Engineering Research and Developments. 39, 83-87 (2016).

10. S.F. Seyed, International Journal of History and Cultural Studies. 2, 6-16 (2016). 